\title{
Growth Mechanism of Cubic-Silicon Carbide Nanowires
}

\author{
K. Y. Cheong and Z. Lockman \\ Electronic Materials Research Group, School of Materials and Mineral Resources Engineering, Engineering Campus, \\ Universiti Sains Malaysia, Nibong Tebal, Penang 14300, Malaysia
}

Correspondence should be addressed to K. Y. Cheong, cheong@eng.usm.my

Received 12 August 2009; Accepted 13 December 2009

Recommended by Donglu Shi

Cubic-SiC nanowires were synthesized using activated carbon powder and Si substrate in vacuum at $1200-1350^{\circ} \mathrm{C}$ for $1-4$ hours. The nanowires were grown according to the following proposed mechanisms: (1) diffusion of C/CO into Si substrate, (2) weakening of $\mathrm{Si}$ bond and atomic kick-out, (3) formation of $\mathrm{Si}-\mathrm{C}$ in vapor phase, (4) formation of saturated $\mathrm{SiC}$ layer, (5) formation of pyramid-like $\mathrm{SiC}$ nanostructure, and (6) formation of SiC nanowires.

Copyright ( $) 2009$ K. Y. Cheong and Z. Lockman. This is an open access article distributed under the Creative Commons Attribution License, which permits unrestricted use, distribution, and reproduction in any medium, provided the original work is properly cited.

\section{Introduction}

$\mathrm{SiC}$ nanowires have gained interest in optoelectronics, high temperature, and high-power applications owing to their excellent chemical, physical, and electronic properties $[1,2]$. Various methods have been used to grow $\mathrm{SiC}$ nanowires, which include laser ablation [3], chemical vapor deposition [4], carbothermal reduction [5], and thermal evaporation process [6]. To explain the formation of the nanowires, three different mechanisms have been proposed, which are vapor-liquid-solid (VLS) growth mechanism $[6,7]$, solid-liquid-solid (SLS) growth mechanism [8], and vaporsolid (VS) growth mechanism [9-12], depending on the configuration of growth/deposition condition and sources of raw materials. Most techniques involved VLS and SLS growth mechanisms, in which a metal catalyst droplet has been used as a nucleation site for adsorption of $\mathrm{SiC}$ vapor phase during the growing process of nanowires [6-8]. However, the nanowires are contaminated by metal residue that is originated from the catalyst [13]. Therefore, some studies have been performed without using any catalyst. Zhang et al. [14] have used silica xerogel and carbon nanoparticles as the source materials to deposit cubic-SiC nanowires on alumina substrate. They were using carbothermal reduction process that performed at Ar ambient. Using the similar set up, Gundiah et al. [5] have replaced the carbon nanoparticles and $\mathrm{Ar}$ gas flow with activated carbon and $\mathrm{NH}_{3}$ or $\mathrm{H}_{2}$ gas, respectively, to produce the same product. The source materials are initially decomposed, reacted and deposited on a substrate, and then nanowires are grown, without consuming any material from the substrate. The production of the cubic-SiC nanowires is according to VS mechanism. In this paper, an alternative route to produce cubic-SiC nanowires is being reported. The nanowires have been grown simultaneously from a Si substrate using an appropriate amount of activated carbon powder under medium vacuum and high temperature conditions. The growth mechanism of the nanowires is totally different from those reported in the literatures.

\section{Experimental}

The growth of $\mathrm{SiC}$ nanowires was performed in a vacuum furnace as schematically outlined in Figure 1. A one surface polished and precleaned n-type $<100>$ oriented Si substrate was used as the Si source and was placed on top of a columniform graphite crucible that contained an appropriate amount of activated carbon powder ( 8.5 at \% of $\mathrm{O}$ and 91.5 at $\%$ of $\mathrm{C})$. The average particle size of the activated carbon powder obtained from a particle size analyzer was $13.55 \mu \mathrm{m}$. To hold the substrate firmly, a graphite block was placed on top of the substrate. The whole setup was placed in the middle of the vacuum furnace that had been evacuated down to $10 \mathrm{~m}$ Torr $\left(1.333 \times 10^{-9} \mathrm{GaP}\right)$. The furnace was then 


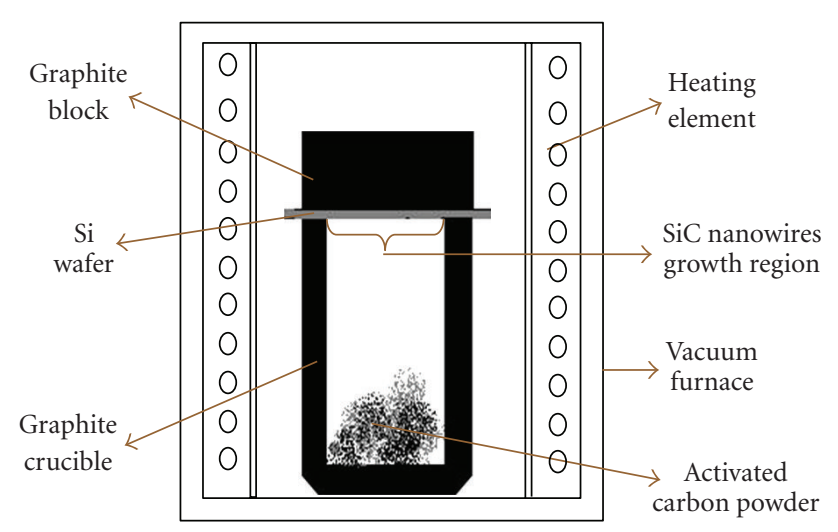

FIgURE 1: Schematic of apparatus setup.

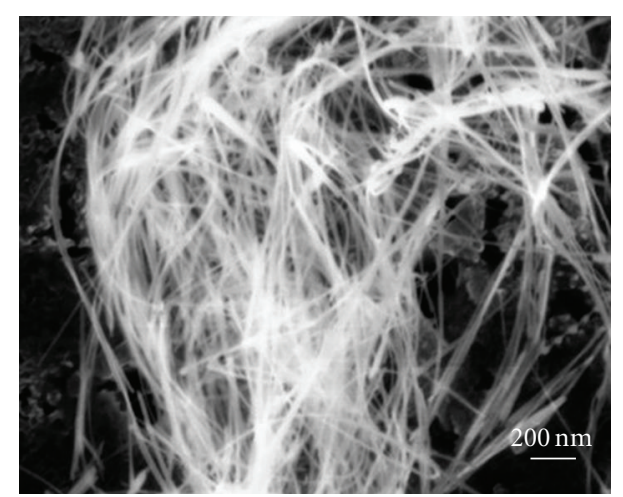

(a)

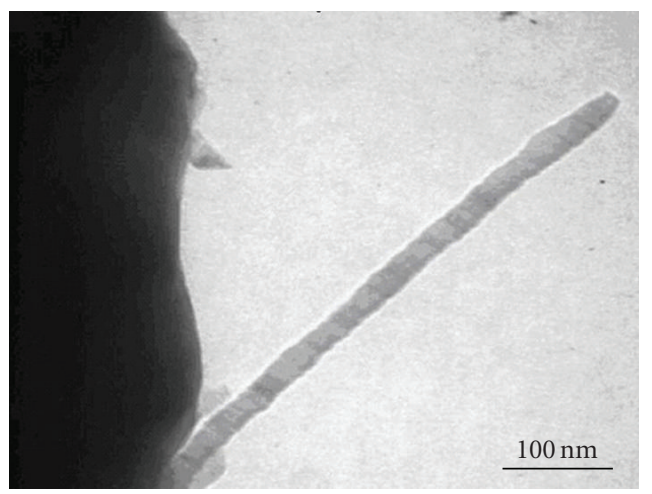

(b)

Figure 2: $3 \mathrm{C}$-SiC nanowires produced at $1300^{\circ} \mathrm{C}$ for $4 \mathrm{~h}$ and observed under (a) FESEM and (b) TEM.

heated up at a rate of $15^{\circ} \mathrm{C} / \mathrm{min}$ to a temperature range of 1200 to $1350^{\circ} \mathrm{C}$ and it was maintained for 1 to 4 hours. After that, the furnace was cooled down $\left(15^{\circ} \mathrm{C} / \mathrm{min}\right)$ in the existing vacuum condition to room temperature. The asgrown $\mathrm{SiC}$ nanowires were characterized by field emission scanning electron microscopy (FESEM) (LEO GEMINI FESEM), X-ray diffraction (XRD) (PANalytical X'pert PRO MRD PW3040), transmission electron microscope (TEM) (Phillips CM12), and Raman spectroscope (Jobin Yvon HR 800 UV).

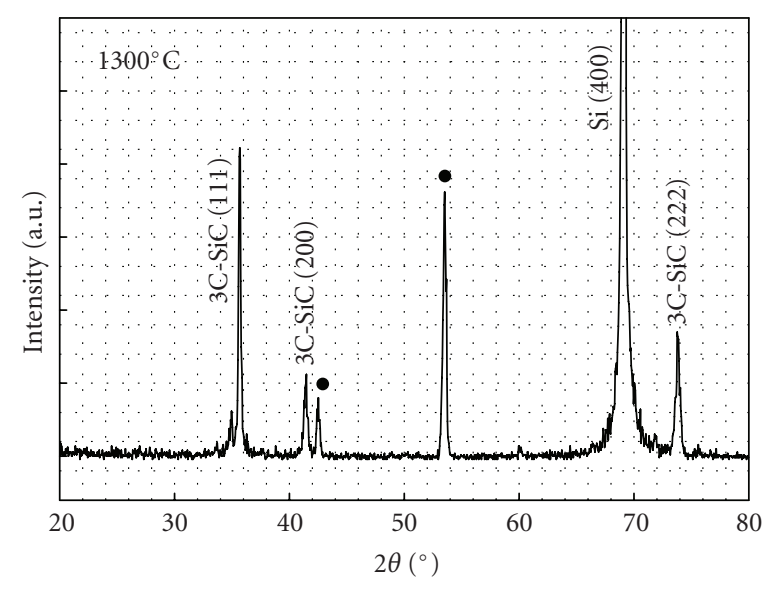

FIGURE 3: XRD spectrum of an as-grown sample produced at $1300^{\circ} \mathrm{C}$ for $4 \mathrm{~h}$. Unidentified phases are indicated by “•”.

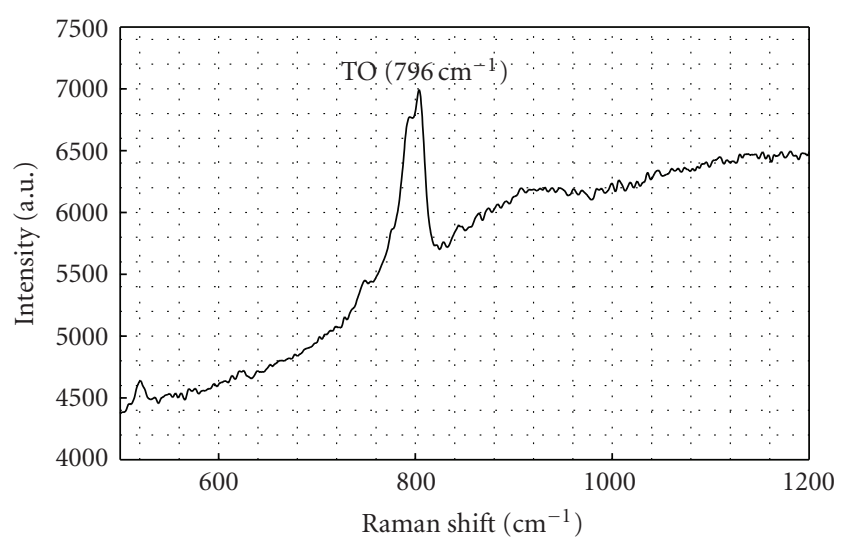

FIgURE 4: Raman spectrum of sample produced at $1300^{\circ} \mathrm{C}$ for $4 \mathrm{~h}$.

\section{Results and Discussions}

Figure 2(a) shows an FESEM micrograph of nanowires growing from clusters of $\mathrm{SiC}$ that have been grown on $\mathrm{Si}$ substrate at $1350^{\circ} \mathrm{C}$ for $4 \mathrm{~h}$. The phases of the nanowires have been investigated by XRD (Figure 3 ) and it has been revealed that the sample contains cubic (3C)-SiC. The $3 \mathrm{C}-$ $\mathrm{SiC}$ peaks appear at $2 \theta=36.6^{\circ}, 41.2^{\circ}$, and $73.9^{\circ}$, which represent the diffraction of $3 \mathrm{C}-\mathrm{SiC}$ (111), (200), and (222) planes, respectively. The obtained values are well matched with JCPDS card no. 29-1129 for the 3C-SiC. Besides $\mathrm{SiC}$ phases, an extremely intense $\mathrm{Si}(400)$ plane has also been detected. This is originated from single crystalline $\mathrm{Si}$ substrate. Due to the high intensity of the single crystal, all of the diffraction peaks have been suppressed. There are additional two diffraction peaks, which are unable to match with either $\mathrm{Si}, \mathrm{SiC}$, or $\mathrm{C}$ phases available in standard JCPDS library. Other than XRD analysis, Raman spectroscopy has also proven that $\mathrm{SiC}$ had been produced using this method (Figure 4). An obvious transverse optical (TO) phonon mode of SiC is recorded at a Raman shift of $796 \mathrm{~cm}^{-1}$ [15].

The nanowires are randomly oriented and tangled together with diameter ranging from 10 to $30 \mathrm{~nm}$ and 
length up to several micrometers (Figure 2(a)). A nanowire is nucleated at a cluster of $\mathrm{SiC}$, which is clearly shown in TEM image presented in Figure 2(b). The production of this nanowire is believed to be governed by six processes that are (1) diffusion of C and/or CO into Si substrate, (2) weakening of Si-Si bond and atomic kick-out, (3) formation of Si-C in vapor phase due to vapor-solid reaction, (4) formation of saturated $\mathrm{SiC}$ layer, (5) formation of pyramid-like $\mathrm{SiC}$ nanostructure due to relaxation of compressive strain at lateral direction, parallel to Si surface, and (6) formation of $\mathrm{SiC}$ nanowires due to relaxation of compressive strain at vertical direction. These processes have been supported by the observation being demonstrated at different growth temperature and time, whereby the transformation of the nanostructures has been proposed.

The diffusion of $\mathrm{C}$ and/or CO into Si surface may be attributed to the following reactions. Carbon atoms initially reacted with a limited amount of oxygen that resided in the activated carbon powder and formed carbon monoxide (CO) gas:

$$
\mathrm{C}_{(\mathrm{s})}+1 / 2 \mathrm{O}_{2(\mathrm{~g})} \longrightarrow \mathrm{CO}_{(\mathrm{g})} .
$$

Based on Boudouard reaction, in this investigation temperature, $\mathrm{CO}_{2(\mathrm{~g})}$ is almost impossible to be formed. It is confirmed that when carbon is reacted with oxygen, $\mathrm{CO}_{(\mathrm{g})}$ is produced. Concurrently, oxygen is also reacted with $\mathrm{Si}$ substrate to for $\mathrm{SiO}_{(\mathrm{g})}$ :

$$
\mathrm{Si}_{(\mathrm{s})}+1 / 2 \mathrm{O}_{2(\mathrm{~g})} \longrightarrow \mathrm{SiO}_{(\mathrm{g})} .
$$

In addition, activated carbon $\left[\mathrm{C}_{(\mathrm{s})}\right]$ may be vaporized $\left[\mathrm{C}_{(\mathrm{g})}\right]$ [16] with the assistance of $\mathrm{CO}$ gas:

$$
\mathrm{C}_{(\mathrm{s})} \longrightarrow \mathrm{C}_{(\mathrm{g})} .
$$

According to the extrapolation from phase diagram of carbon, at medium high vacuum and high-temperature condition, carbon in activated form can be transformed into gas phase [17]. Due to existence of vacuum in the furnace and difference in pressure inside and outside of the crucible, emission of $\mathrm{CO}$ and/or $\mathrm{C}$ gaseous may drift upward. As a result, $\mathrm{CO}_{(\mathrm{g})}$ and/or $\mathrm{C}_{(\mathrm{g})}$ may diffuse towards surface of the $\mathrm{Si}$ substrate. The $\mathrm{C}_{(\mathrm{g})}$ may directly react with $\mathrm{Si}$ substrate and form $\mathrm{SiC}$ :

$$
\mathrm{Si}_{(\mathrm{s})}+\mathrm{C}_{(\mathrm{g})} \longrightarrow \mathrm{SiC}_{(\mathrm{s})} .
$$

Simultaneously, $\mathrm{CO}_{(\mathrm{g})}$ may also react with the Si substrate in a vapor-solid reaction:

$$
\mathrm{C}_{(\mathrm{g})}+2 \mathrm{Si}_{(\mathrm{s})}+\mathrm{CO}_{(\mathrm{g})} \longrightarrow 2 \mathrm{SiC}_{(\mathrm{s})}+\mathrm{O}_{(\mathrm{g})} .
$$

The proposed reactions (4) and (5) are responsible for the nucleation of $\mathrm{SiC}$. In order for the nanowires to grow, reaction (6) may take place,

$$
\mathrm{SiO}_{(\mathrm{g})}+\mathrm{CO}_{(\mathrm{g})} \longrightarrow \mathrm{SiC}_{(\mathrm{s})}+\mathrm{O}_{2(\mathrm{~g})} .
$$

The by-product $\left[\mathrm{O}_{(\mathrm{g})}\right]$ of reactions (5) and (6) is then utilized to react with the activated carbon $\left[\mathrm{C}_{(\mathrm{s})}\right]$ as shown in (1) and the whole processes are repeated.

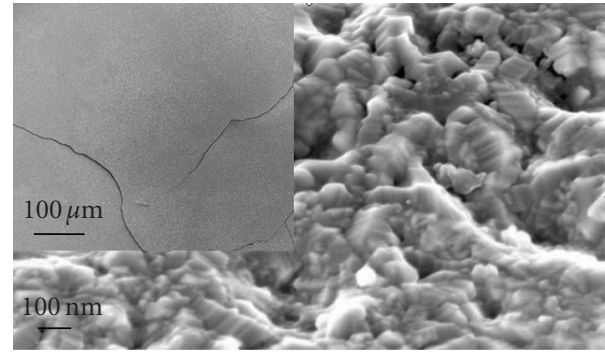

(a)

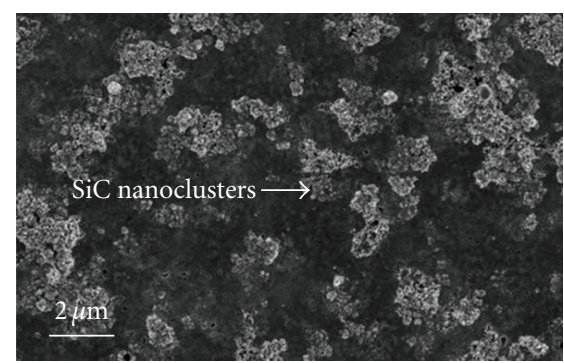

(b)

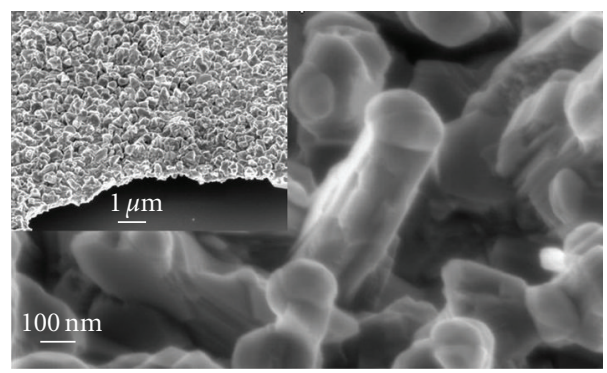

(c)

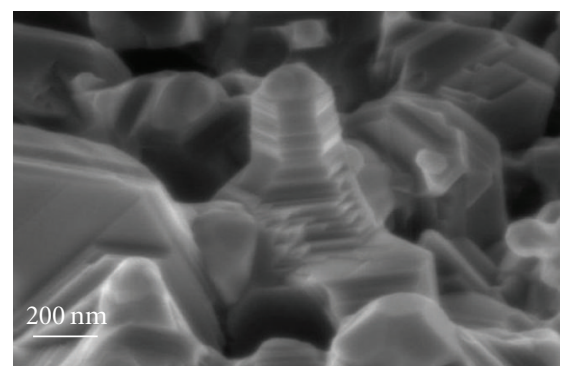

(d)

FIGURE 5: FESEM micrographs of Si surface heated for $1 \mathrm{~h}$ at different temperatures with activated carbon powder. (a) Porous Si structures are observed at $1200^{\circ} \mathrm{C}$ with cracks shown in the inset of the figure. (b) Nanoclusters of $\mathrm{SiC}$ are revealed at $1250^{\circ} \mathrm{C}$, (c) Spherical and faceted $\mathrm{SiC}$ structures are shown at $1300^{\circ} \mathrm{C}$ (inset: layers of $\mathrm{SiC}$ on the Si substrate), and (d) Pyramid-like SiC structures are demonstrated at $1350^{\circ} \mathrm{C}$.

As the growth temperature increases, bonding of $\mathrm{Si}-\mathrm{Si}$ in the $\mathrm{Si}$ substrate may be weakened; eventually the $\mathrm{Si}-\mathrm{Si}$ bonds are dissociated and Si atoms, in gas phase, are released. This process may be enhanced by kick-out effect due to indiffusion of $\mathrm{CO}$ and/or C gaseous [18]. The in-diffusion rate is increased as the growth temperature increases. The 
difference in molecular or atomic diameter of $\mathrm{CO}$ or $\mathrm{C}$ with respect to $\mathrm{Si}$ may induce a volume change and lattice strain to the surrounding atoms. Therefore, a higher strain is produced in the Si lattice that ultimately may lead to crack formation on the Si surface. The dissociation of Si-Si bonds and generation of cracks (inset of Figure 5(a)) forms a very porous Si surface (Figure 5(a)), suggesting that $\mathrm{Si}$ atoms have been removed [9]. The removed $\mathrm{Si}$ atoms in gas phase may react with $\mathrm{CO}$ and/or $\mathrm{C}$ to form $\mathrm{SiC}$ molecules and subsequently the molecules may be deposited on the porous Si surface as small clusters. Figure 5(b) reveals the small clusters of SiC formed at $1250^{\circ} \mathrm{C}$ for $1 \mathrm{~h}$. At higher temperature $\left(1300^{\circ} \mathrm{C}\right)$, while maintaining the time, layers of $\mathrm{SiC}$ are formed (inset of Figure 5(c)) on Si substrate due to a higher reaction rate between $\mathrm{Si}$ and $\mathrm{C}$ as well as $\mathrm{CO}$. This reaction is enhanced by the temperature as more carbon sources are able to diffuse deeper inside the Si lattice through the cracks that have been generated earlier. The SiC layers consist of spherical and faceted structures (Figure 5(c)). The formation of this structure may be due to the following explanation.

The molecular size of $\mathrm{SiC}$ is larger as compared to $\mathrm{Si}$ and $\mathrm{C}$ atoms. Thus, the equilibrium structure of Si lattice is disturbed when $\mathrm{SiC}$ is formed and it may induce a compressive strain onto the surrounding atoms. The strain needs to be released in order to achieve an equilibrium condition in the system. The relaxation may be achieved by releasing additional energy (in terms of force) in lateral (which is parallel to Si surface) and/or vertical directions. Since the releasing of energy is easier in lateral direction than in vertical direction, therefore, the lateral force should be larger than the vertical force. Consequently, a layer of $\mathrm{SiC}$ is formed laterally. Until certain extent, when the compressive strain is so high that it cannot be released through the lateral or vertical force, then cracks are generated on the surface of the $\mathrm{SiC}$ layers.

As the temperature is further increased $\left(1350^{\circ} \mathrm{C}\right)$, carbon sources may further diffuse deeper into the cracked $\mathrm{Si}$ substrate and $\mathrm{SiC}$ layers so that it could further be reacted with $\mathrm{Si}$ to form more SiC. Since the surface of the Si has been transformed into $\mathrm{SiC}$, the only path for the carbon-related source to diffuse deeper inside is through the cracks. In order for the carbon-related source to form $\mathrm{SiC}$, Si source must be available and the only $\mathrm{Si}$ available source is from $\mathrm{Si}$ substrate. If the carbon-related source is only interact with the initial layer of $\mathrm{SiC}$ surface, which has been formed on top of the Si substrate, and not diffused into the crack, most probably deposition of carbon is observed rather than growing of SiC. However, in this work, it has been proven that $\mathrm{SiC}$ nanowires have been produced and, therefore, the production of the nanowire is via a growing process and not a deposition process. The difference in molecular size of $\mathrm{SiC}$ and atomic size of Si may again cause a redistribution of the atoms and molecules in the lattice laterally and/or vertically, so that the induced strain can be relaxed. The spherical and faceted $\mathrm{SiC}$ layer structures are now transformed into pyramidlike structures with stacked SiC layers (Figure 5(d)). The formation of this structure is considered a localized epitaxial growth [19]. When the growth time is prolonged, from $1 \mathrm{~h}$

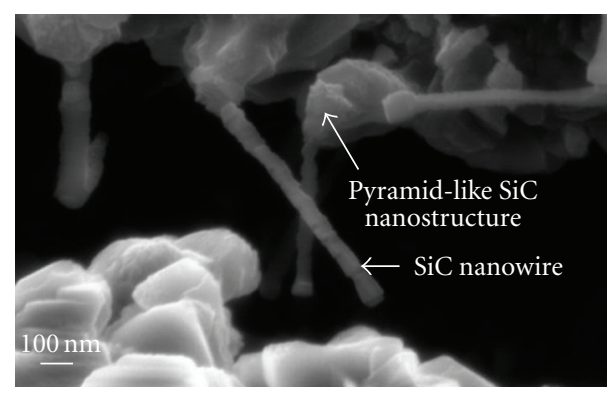

Figure 6: FESEM micrograph 3C-SiC nanowires originated from pyramid-like SiC structures.

to $4 \mathrm{~h}, \mathrm{SiC}$ nanowires started to form (Figure $2(\mathrm{a})$ ). The nanowires are originated from the tip of pyramid structures as revealed in Figure 6. The transformation of pyramidlike $\mathrm{SiC}$ nanostructures into nanowires is probably due to the relaxation of compressive strain at vertical direction as it is much easier if compared to at lateral direction. The relaxation of compressive strain at vertical direction may induce larger vertical force. Consequently, nanowires are protruded from the pyramid structure and elongated vertically.

\section{Conclusions}

The growth mechanism of $3 \mathrm{C}-\mathrm{SiC}$ nanowires was proposed based on the results obtained from a heated Si substrate and activated carbon power in vacuum at 1200 to $1350^{\circ} \mathrm{C}$ for 1 to $4 \mathrm{~h}$. Six different processes were responsible for the formation of the nanowires, namely, (1) diffusion of $\mathrm{C}$ and/or CO into Si substrate, (2) weakening of Si-Si bond and atomic kick-out, (3) formation of $\mathrm{Si}-\mathrm{C}$ in vapor phase due to vapor-solid reaction, (4) formation of saturated $\mathrm{SiC}$ layer, (5) formation of pyramid-like $\mathrm{SiC}$ nanostructure due to relaxation of compressive strain at lateral direction, and (6) formation of $\mathrm{SiC}$ nanowires due to relaxation of compressive strain at vertical direction.

\section{Acknowledgment}

This material is based upon work supported by the Malaysian Toray Science Foundation (MTSF-6050114).

\section{References}

[1] H. Matsunami, "Current SiC technology for power electronic devices beyond Si," Microelectronic Engineering, vol. 83, no. 1, pp. 2-4, 2006.

[2] A. R. Powell and L. B. Rowland, "SiC materials-progress, status, and potential roadblocks," Proceedings of the IEEE, vol. 90, no. 6, pp. 942-955, 2002.

[3] W. Shi, Y. Zheng, H. Peng, N. Wang, C. S. Lee, and S.-T. Lee, "Laser ablation synthesis and optical characterization of silicon carbide nanowires," Journal of the American Ceramic Society, vol. 83, no. 12, pp. 3228-3230, 2000. 
[4] H.-K. Seong, H.-J. Choi, S.-K. Lee, J.-I. Lee, and D.-J. Choi, "Optical and electrical transport properties in silicon carbide nanowires," Applied Physics Letters, vol. 85, no. 7, pp. 12561258, 2004.

[5] G. Gundiah, G. V. Madhav, A. Govindaraj, M. M. Seikh, and C. N. R. Rao, "Synthesis and characterization of silicon carbide, silicon oxynitride and silicon nitride nanowires," Journal of Materials Chemistry, vol. 12, no. 5, pp. 1606-1611, 2002.

[6] G. Shen, Y. Bando, C. Ye, B. Liu, and D. Golberg, "Synthesis, characterization and field-emission properties of bamboo-like $\beta$-SiC nanowires," Nanotechnology, vol. 17, no. 14, pp. 34683472, 2006.

[7] D. H. Feng, T. Q. Jia, X. X. Li, et al., "Catalytic synthesis and photoluminescence of needle-shaped 3C-SiC nanowires," Solid State Communications, vol. 128, no. 8, pp. 295-297, 2003.

[8] Y. Baek, Y. Ryu, and K. Yong, "Structural characterization of $\beta$-SiC nanowires synthesized by direct heating method," Materials Science and Engineering C, vol. 26, no. 5-7, pp. 805808, 2006.

[9] X. W. Du, X. Zhao, S. L. Jia, Y. W. Lu, J. J. Li, and N. Q. Zhao, "Direct synthesis of $\mathrm{SiC}$ nanowires by multiple reaction VS growth," Materials Science and Engineering B, vol. 136, no. 1, pp. 72-77, 2007.

[10] J. Chen, R. Wu, G. Yang, et al., "Synthesis and photoluminescence of needle-shaped 3C-SiC nanowires on the substrate of PAN carbon fiber," Journal of Alloys and Compounds, vol. 456, no. 1-2, pp. 320-323, 2008.

[11] J. J. Niu and J. N. Wang, "A simple route to synthesize scales of aligned single-crystalline $\mathrm{SiC}$ nanowires arrays with very small diameter and optical properties," Journal of Physical Chemistry $B$, vol. 111, no. 17 , pp. 4368-4373, 2007.

[12] J. J. Niu, J. N. Wang, and Q. F. Xu, "Aligned silicon carbide nanowire crossed nets with high superhydrophobicity," Langmuir, vol. 24, no. 13, pp. 6918-6923, 2008.

[13] K. Saulig-Wenger, D. Cornu, F. Chassagneux, G. Ferro, T. Epicier, and P. Miele, "Direct synthesis of $\beta$-SiC and h-BN coated $\beta$-SiC nanowires," Solid State Communications, vol. 124, no. 4, pp. 157-161, 2002.

[14] L. D. Zhang, G. W. Meng, and F. Phillipp, "Synthesis and characterization of nanowires and nanocables," Materials Science and Engineering A, vol. 286, no. 1, pp. 34-38, 2000.

[15] W. N. F. W. Mustapha, K. Y. Cheong, and Z. Lockman, "Synthesis of R-SiC nanowires via catalyst-free chemical vapour growth route," in Proceedings of the 2nd IEEE International Nanoelectronics Conference (INEC '08), pp. 438-442, Shanghai, China, March 2008.

[16] A. I. Kharlamov, N. V. Kirillova, L. A. Karachevtseva, and A. A. Kharlamova, "Low-temperature reactions between vaporizing silicon and carbon," Theoretical and Experimental Chemistry, vol. 39, no. 6, pp. 374-379, 2003.

[17] L. M. Ghiringhelli, C. Valeriani, J. H. Los, E. J. Meijer, A. Fasolino, and D. Frenkel, "State-of-the-art models for the phase diagram of carbon and diamond nucleation," Molecular Physics, vol. 106, no. 16-18, pp. 2011-2038, 2008.

[18] T. Y. Tan and U. Gösele, "Diffusion in semiconductors," in Diffusion in Condensed Matter, P. Heitjans and J. Kärger, Eds., pp. 165-208, Springer, Berlin, Germany, 2005.

[19] D. Moore, Ph.D. thesis, Georgia Institute of Technology, Atlanta, Ga, USA, 2006. 

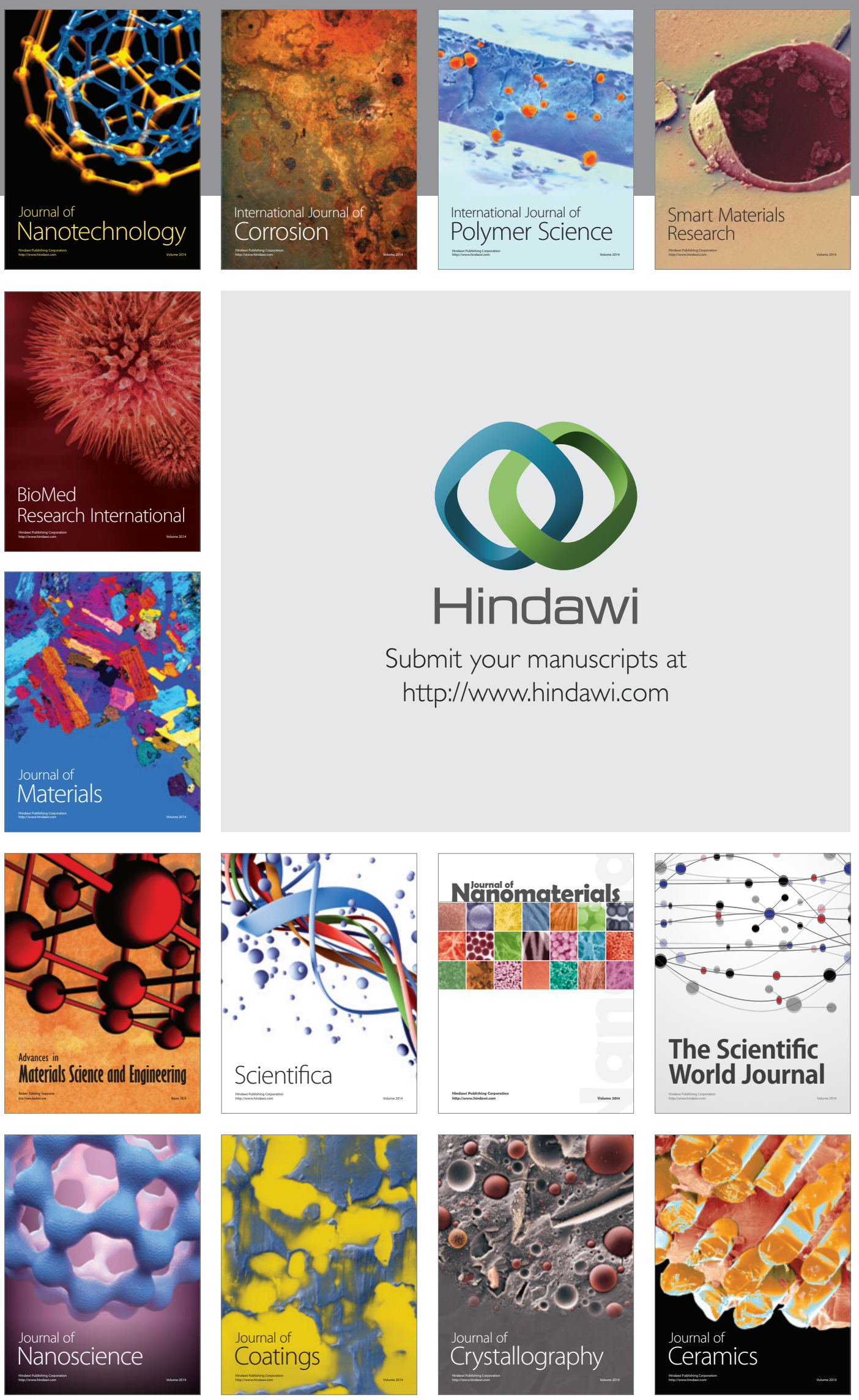

The Scientific World Journal

Submit your manuscripts at

http://www.hindawi.com

\section{World Journal}

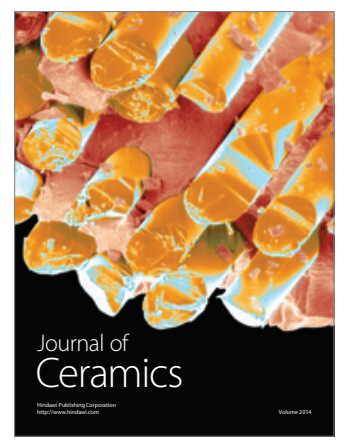

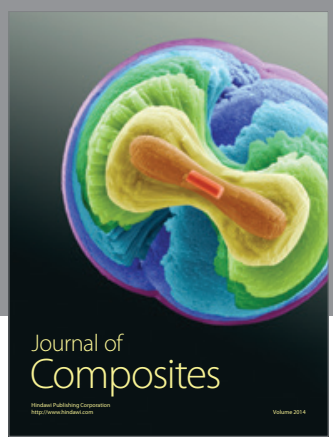
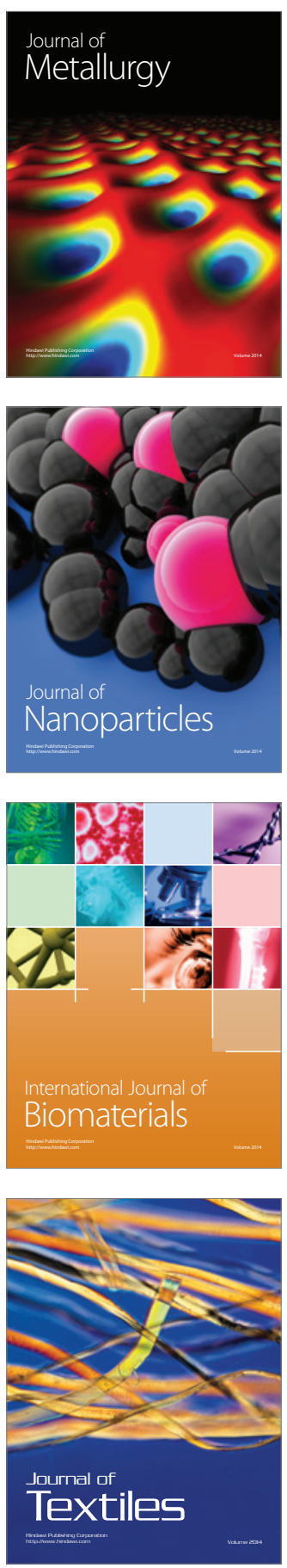